\title{
HIGH QE PHOTOCATHODES PERFORMANCE DURING OPERATION AT FLASH / PITZ PHOTOINJECTORS*
}

\author{
L. Monaco ${ }^{\#}$, P. Michelato, C. Pagani, D. Sertore, \\ INFN Milano - LASA, I-20090 Segrate (MI), Italy \\ J. W. Bähr, J. H. Han, M. Krasilnikov, S. Lederer, S. Schreiber, F. Stephan, \\ DESY, D-22603 Hamburg, Germany
}

\begin{abstract}
Since 1998, the DESY photoinjectors at FLASH and PITZ routinely use high quantum efficiency (QE) photocathodes produced at LASA (INFN-Milano). To further understand the photocathode behavior during beam operation, photocathode QE measurements have been performed at different operating conditions in both photoinjectors. The analysis of these measurements will be used to improve the photocathode preparation procedures and deepen our understanding of the photocathode properties. An important goal is to further increase their lifetime and to preserve a high beam quality during the operation in the RF gun of the photoinjector.
\end{abstract}

\section{INTRODUCTION}

Since the 90s, INFN Milano is involved in the study of the growth process of photocathodes based on alkali telluride [1]. Due to this experience, in 1998 INFN Milano started the production of photocathodes for TTF/FLASH and later, from 2000, the production of photocathodes for the PITZ facility [2]. FLASH is the free electron laser at DESY operating since 2005 as a user facility, PITZ at DESY Zeuthen is a test stand for photoinjector developments.

Up to now, we have produced 57 photocathodes $\left(\mathrm{Cs}_{2} \mathrm{Te}\right.$ and KCsTe films and Mo plugs without coatings). The production process has been optimized during the years applying both the knowledge gathered in our R\&D activities and the experience achieved in the photocathode production process and in the cathode usage in RF guns. Moreover, all data related to the main steps of the production process and also to the cathode performances during gun operation are retrieved and organized in a dedicated web-accessible database [3].

Figures of merit for the photocathode characterization are the operative lifetime, the achievable current density, the extracted charge, dark current, the sensitivity to gas exposition, the quantum efficiency (QE), and the uniformity of the sensitive layer. The QE or the cathode uniformity, are usually monitored during the growth of the photoemissive films, before their delivery, and after their usage in the RF guns. The latter is to understand their robustness and to investigate damages of the photoemissive film due operation with high accelerating gradients. Other aspects are mainly checked during

\footnotetext{
* Work partially supported by the European Community, contract numbers RII3-CT-2004-506008.

\#laura.monaco@mi.infn.it
}

cathode operation, such as the dark current [4] or the extracted charge [5]. Up to now, none of the cathodes has shown any limitation in the extracted charge, even after long periods of usage. Typically, the main reason to change cathodes during the gun operation is a growth of dark current to high values, tests of other cathode types, or a reduction of quantum efficiency below $1 \%$.

Both at FLASH and at PITZ, the quantum efficiency is measured routinely: monitoring the cathode lifetime is essential to optimize the uptime of the photoinjector.

Also the photoemissive surface response uniformity is an important information. A so called QE map is routinely created at LASA after their production and, from time to time, in situ in the RF gun. The uniformity affects the beam quality and thus the performance of the SASE lasing process at FLASH.

In this paper, the history of several cathodes which have been in operation since June 2006 is discussed. Emphasis is on their QE and uniformity from the production phase to their usage.

\section{PHOTOCATHODE PRODUCTION}

Table 1 summarizes the main characteristic of $\mathrm{Cs}_{2} \mathrm{Te}$ photocathodes produced and considered in this paper. The standard recipe [2] has been used for their production. The only difference respect to the usual procedure is in the Mo cleaning. Plug 34 has been treated, besides the standard procedure also with $\mathrm{CO}_{2}$ at DESY.

Table 1: Photocathode main characteristics.

\begin{tabular}{|c|c|c|c|c|c|}
\hline Cathode & $\mathbf{R}^{\#}(\%)$ & Dep.(date) & $\begin{array}{l}\text { Te } \\
\text { (nm) }\end{array}$ & $\begin{array}{l}\text { QE(\%) } \\
\text { @ 254nm }\end{array}$ & $\begin{array}{l}\mathrm{QE}(\%) \\
\text { @ 262nm }\end{array}$ \\
\hline $78.1^{£}$ & 56.2 & Apr. 5, '06 & 10 & 7.7 & 6.1 \\
\hline 77.1 & 56.5 & Apr.4, ‘06 & 10 & 7.2 & 5.2 \\
\hline 95.1 & 56.4 & Sep. 13, ‘06 & 10 & 12.2 & 7.8 \\
\hline 13.3 & 56.0 & Sep. 12, ‘06 & 10 & 14.9 & 10.0 \\
\hline 92.1 & 56.4 & Sep. 15, ‘06 & 15 & 11.6 & 7.7 \\
\hline 94.1 & 56.4 & Sep. 14, '06 & 15 & 12.8 & 8.5 \\
\hline $34.6^{\S}$ & 56.5 & Dec. 15, '06 & 10 & 11.5 & 7.5 \\
\hline
\end{tabular}

${ }^{\varepsilon}$ Electro-polished before the optical polishing treatment

$\S$ Standard cleaning (Acetone + Alcohol) $+\mathrm{CO}_{2}$ cleaning

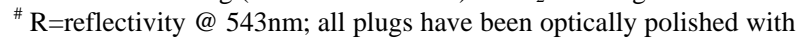
the "automatic" technique [2]

The cathode growth is qualified collecting the emitted photocurrent by illuminating the cathode with an $\mathrm{Hg}$ lamp. Just after the deposition of the film, we measure the 
spectral response and uniformity. Several wavelength around $254 \mathrm{~nm}$ are used. Fig. 1 shows the measured spectral response of $\mathrm{Cs}_{2} \mathrm{Te}$ cathodes just after their deposition. The response at photoinjector lasers wavelength $(\lambda=262 \mathrm{~nm})$ is obtained by interpolation.

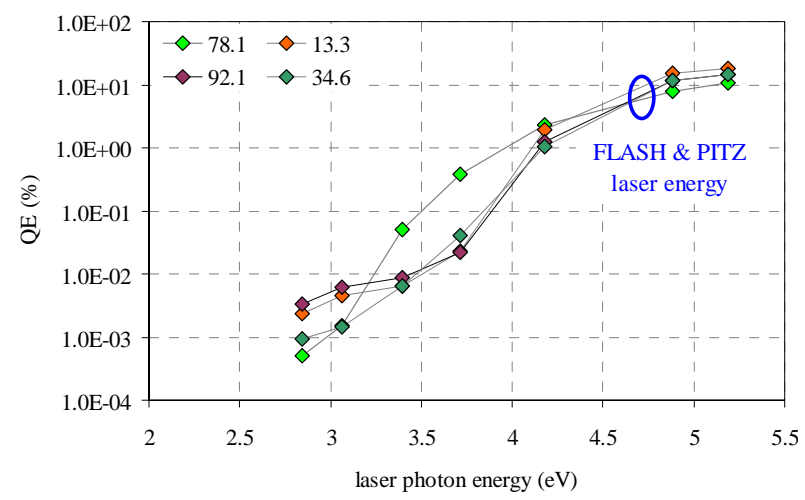

Fig. 1: Spectral response of cathodes 78.1, 13.3, 92.1 and 34.1 just after their deposition.

A typical QE map is shown in Fig. 2 (left), measured using $\lambda=254 \mathrm{~nm}$ with a spot of $1 \mathrm{~mm}$ in diameter. In the same figure (right) a photo shows the same cathode in the transport box before the delivery.
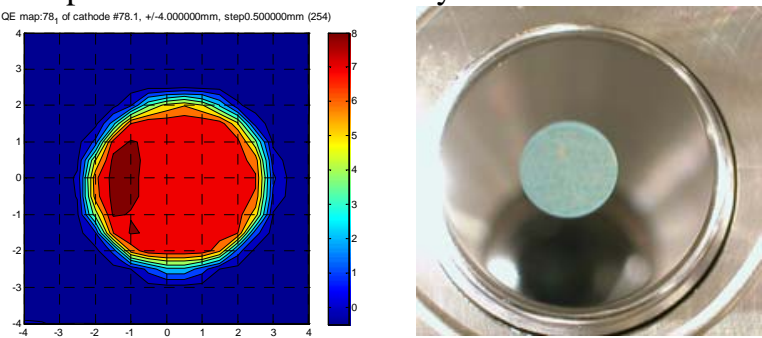

Fig. 2: QE map at 254nm (left) and a photo (right) of cathode 78.1 just after its deposition.

\section{FLASH DATA}

At FLASH, an automated procedure has been set-up to frequently monitor the QE during operation [6]. The emitted charge is measured as a function of the laser energy. A calibrated toroid $( \pm 1 \%)$ and a calibrated joulemeter (Molectron $\pm 5 \%$ ) are used. The charge increases linearly with the laser energy until space charge effects lead to its saturation (see Fig. 3).

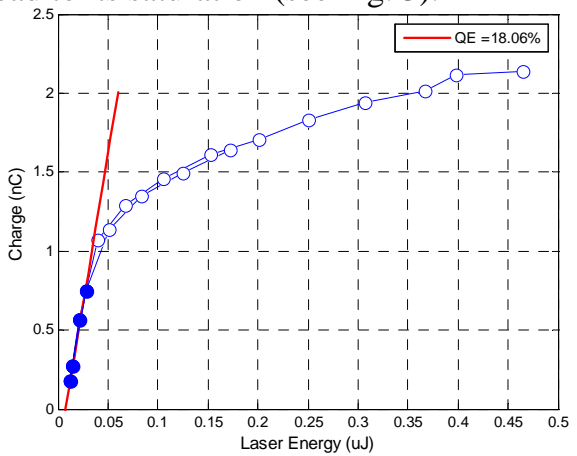

Fig. 3: QE of cathode 13.3 measured Nov. 3, 2006, FLASH RF gun. Laser wavelength: $262 \mathrm{~nm}$. A fit to the linear part gives $18 \%$.
The QE is calculated from a straight line fit to the linear part. The statistical and systematic errors of the measurements presented here are in the order of $20 \%$. The systematic error is mainly due to the uncertainty of identifying the linear part for the fit and due to the crosscalibration of the laser pulse energy monitor with the energy measured in front of the vacuum window.

Figure 4 shows the summary of QE measurements at the FLASH RF gun for several cathodes. The data are taken since April 2006, always under the same operative conditions. An initial fast drop of the quantum efficiency is observed. Most cathodes show a stabilization of the QE below $5 \%$.

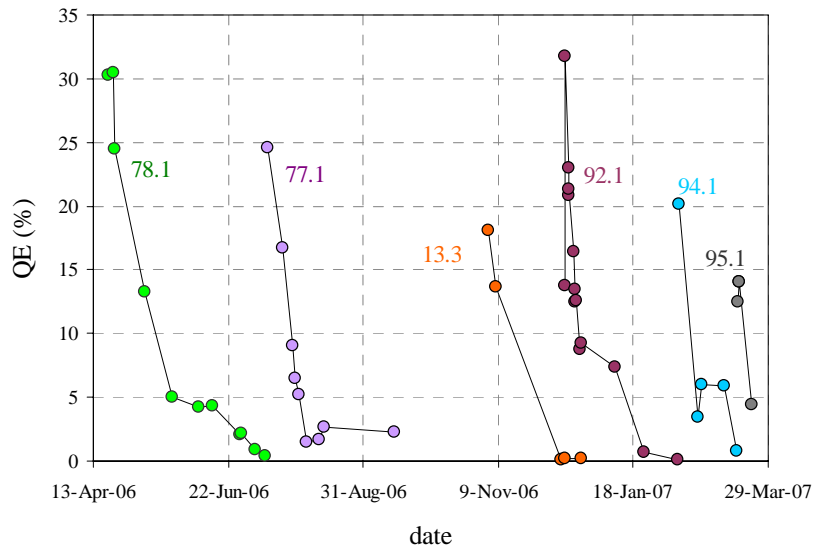

Fig. 4: Summary of QE measurements at the FLASH for several cathodes. Measurement conditions: forward power: $3.2 \mathrm{MW}\left(\mathrm{E}_{\mathrm{acc}}=42 \mathrm{MV} / \mathrm{m}\right)$, phase gun $\mathrm{RF} / \mathrm{laser}$ pulses: 38 deg off zero crossing.

Table 2 summarizes the operative lifetime of the cathodes as shown in Fig. 4. The cathode is considered to be at the end of its lifetimes when the QE decreases below $0.5 \%$. All cathodes are used $24 \mathrm{~h}$ a day and 7 days per week.

Table 2: Photocathode operation lifetime at FLASH.

\begin{tabular}{|l|c|c|c|c|c|c|}
\hline Cathode & $\mathbf{7 8 . 1}$ & $\mathbf{7 7 . 1}$ & $\mathbf{9 5 . 1}$ & $\mathbf{1 3 . 3}$ & $\mathbf{9 2 . 1}$ & $\mathbf{9 4 . 1}$ \\
\hline Op. lifetime (days) & 83 & 85 & $22^{\&}$ & 40 & 39 & 34 \\
\hline
\end{tabular}
\& until shutdown March 2007

The cathodes discussed here show a shorter operative lifetime than previous cathodes [5]. We do not yet have a convincing explanation. The most probable reason for the shorter lifetime is the observed increase of the vacuum pressure by an order of magnitude during operation with long RF pulses and laser pulse trains (800 $\mu$ s) [4]. Another source of degradation could be a damaging of the cathode surface due to ion back bombardment. QE maps and photos of cathodes during their operation in the gun show areas where the visible color has changed. Fig. 5 shows two QE maps obtained at FLASH of cathode 78.1. The first map has been obtained June 6 when the cathode was fresh, the second map after 7 weeks (July 25). While the fresh cathode has a uniform QE map, the picture changed after a couple of weeks of usage: the central part has a reduced QE, where the rims of the cathode kept the 
initial QE. A visual inspection reveals a change in color of the low QE area to dark blue. After transfer to LASA (keeping UH-vacuum), we have confirmed the shape and that the darker area in the photograph coincides with the low QE area. It is interesting to note, that the size of the low QE area is roughly the size of the laser beam. The laser is centered to the electrical center of the rf gun, the cathode film itself may not be concentric with the field. This could explain the shift of the laser beam to the side in respect to the cathode film.

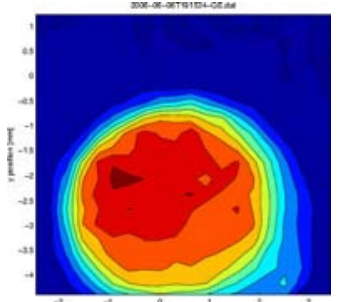

June 6, '06 (FLASH)

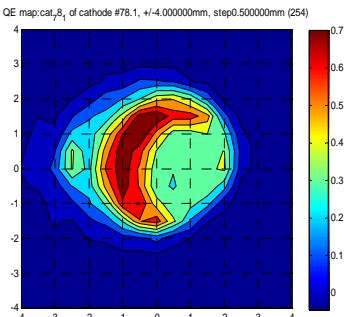

May 4, '07 (LASA)

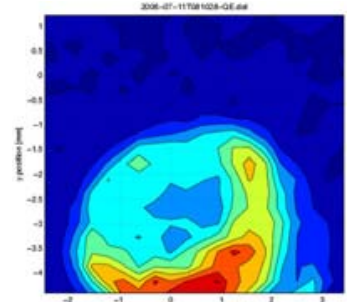

July 25, ’06 (FLASH)

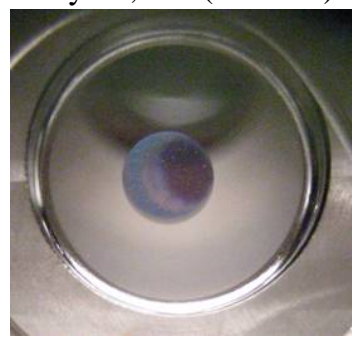

May 4, '07 (LASA)
Fig. 5: Cathode 78.1. Upper left, QE map of the fresh cathode at FLASH. Upper right, QE map after usage, lower left, QE map measured afterwards at LASA and (lower right) a photograph keeping the orientation.

\section{PITZ DATA}

At PITZ, the experimental program emphasis the operation at very high accelerating fields. Field gradients of up to $60 \mathrm{MV} / \mathrm{m}$ on the cathode surface are obtained (Lband RF gun at $1.3 \mathrm{GHz}$ )

Fig. 6 shows a QE measurement of cathode 34.6 at $60 \mathrm{MV} / \mathrm{m}$. The charge is measured as a function of the laser energy and the linear part fit gives a QE of $4.6 \%$.

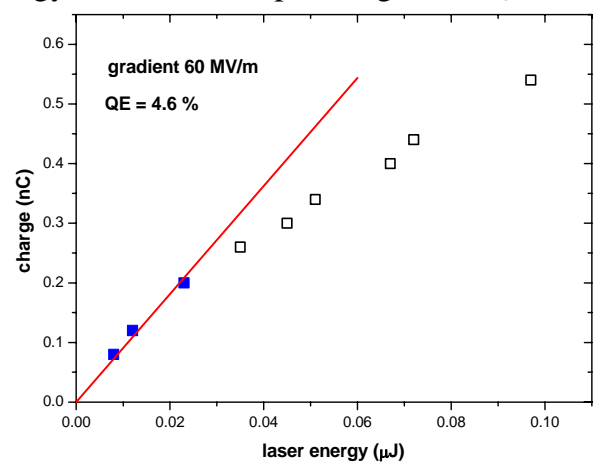

Fig. 6: QE of cathode 34.6 measured May 30, 2007 in the PITZ RF-gun. Laser wavelength: $262 \mathrm{~nm}$.

To study the dependence of the QE on the accelerating gradient, Fig. 7 shows the measured QE for 3 accelerating gradients on the same cathode. The relative phase
laser/RF is taken into account to evaluate the gradient on the cathode surface. The extracted charge increases with the accelerating gradient due to the Schottky effect.

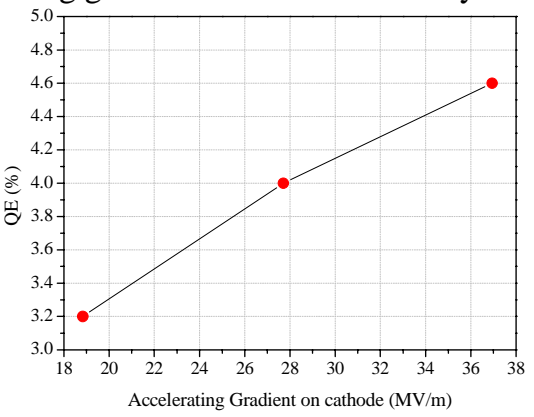

Fig. 7: QE for different accelerating gradients on the cathode surface.

At PITZ, QE maps are realized only recently. A first attempt for such a measurement is shown in Fig. 8, where some data are missing due to problem in the control program of the wedge plates (i.e. the vertical row visible in the picture). The black points are absolute QE measurements at five positions obtained with the same laser spot size on the cathode, as during the mapping. No QE deterioration as at FLASH has been observed yet.

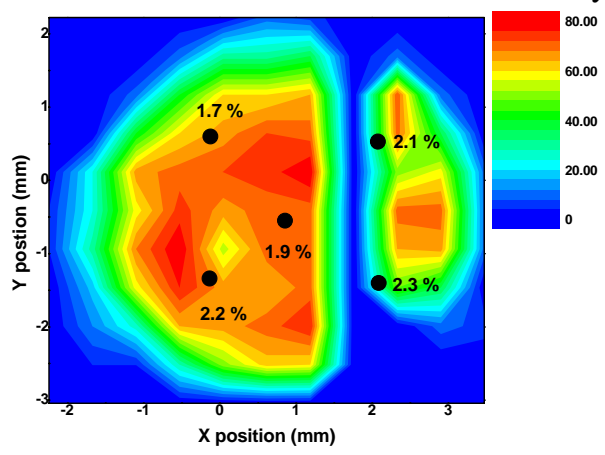

Fig. 8: Cathode 34.6 QE map at PITZ $(\lambda=262 \mathrm{~nm})$.

\section{CONCLUSION}

The performances of the photocathodes used at FLASH/PITZ since June 2006 have been reviewed. Analysis of the QE vs. time and $\mathrm{E}_{\mathrm{acc}}$, together with $\mathrm{QE}$ maps in function of the operative time has been discussed. Measurements at FLASH and at LASA highlights that local no-uniformity are correlated to the color changes of photoemissive films. Results so far obtained encourage further improvements of these tools useful for online diagnostic of the cathode during their operation.

\section{REFERENCES}

[1] P. Michelato et al., NIM. A, 393 (1997) 464.

[2] D. Sertore et al., MOPB009, PAC05, Knoxville, Tennessee, USA, 2005.

[3] http://wwwlasa.mi.infn.it/ttfcathodes/

[4] L. Monaco et al., WEPLS051, EPAC06, Edinburgh, UK, 2006.

[5] D. Sertore et al., WEPLS052, EPAC06, Edinburgh, UK, 2006.

[6] D. Sertore et al., TUPMA083, APAC2007. 